Scientific Visualization, 2020, volume 12, number 2, pages 53 - 73, DOI: 10.26583/sv.12.2.05

\title{
Visual cognitive control of space systems radiotechnical signals
}

\author{
Yu.G. Emelyanova1,A, M.V. Khachumov²,B,C \\ A PSI RAS (Ailamazyan Program Systems Institute of RAS) \\ B FRC CSC RAS (Federal Research Center "Computer Science and Control" \\ of Russian Academy of Sciences) \\ c RUDN University (Peoples Friendship University of Russia) \\ 1. ORCID: 0ooo-ooo1-7735-6820, yuliya.emelyanowa2015@yandex.ru \\ 2 ORCID: 000o-0001-5117-384X, khmike@inbox.ru
}

\begin{abstract}
The method of cognitive graphical information presentation is developed, allowing to classify radiotechnical signals and estimate the degree of noise. The method is based on the construction of a features set ordered by information significance. In turn, informativeness is determined by the formal contribution of the feature to the quality of signal type recognition. The construction of cognitive images is carried out in several stages: 1) determination of numerical characteristics of typical signals, 2) ranking and selection of the most informative characteristics, 3) construction of cognitive graphic images visualizing a multidimensional vector of signal features, 4) operator's interpretation of cognitive images.

The method of integral contour representation polar scan is used to construct cognitivegraphic images of signals. A total of forty informative parameters of the signal (features) are calculated. To increase the polar scan selectivity, the features are ranked in informativeness descending order by the Add and Del methods. The operation of signals subtraction defined over their informative parameters is introduced. With the goal to improve the visual recognition quality, monochrome halftones have been added to cognitive images. To improve perception of the contour representation of difference images color components have been introduced. The sensitivity of the cognitive images to substantial noise signals is expressed through changes in polar scan forms, tones and colorful presentation. The comparison of signals recognition quality by using metrics and polar scan visual recognition is provided. The recommendations are given to the decision-making operator on the type and noise degree of the radio signal in the final part.
\end{abstract}

Keywords: radiotechnical signal, characteristics environment, information importance, recognition, cognitive graphics, cognitive image, multidimensional data visualization, analysis of radiotechnical signals, interpretation of a cognitive image.

\section{Introduction}

One of the essential tasks of processing electronic signals coming from space systems is their operational analysis in order to detect abnormal situations and support decision-making by a human operator [1-3]. New man-machine interaction is based on the technologies of figurative presentation and interpretation of large volumes of information that contribute to quick decision-making. Cognitive data visualization is a promising area, complementing the means of monitoring and diagnostics of the interface of command and instrumentation systems (GS CIS). However, at present, there are no uniform principles for constructing cognitive images that can carry large volumes and vast flows of information in generalized, visual, efficiently, and reliably perceived forms for users. As a rule, images are created individually taking into 
account a specific application area and are interpreted by an expert based on learning and accumulated knowledge [4].

The development of cognitive graphics tools has begun relatively recently. One of the earliest definitions of cognitive graphics, in particular, is given in [5] concerning medical topics: "Cognitive graphics are a set of visual representations that help conclude on complex cognitive problems such as diagnosis and monitoring." In Russian-language literature, the term "cognitive graphics" (CG) was first introduced by A. Zenkin. By his definition, "cognitive graphics" is a combination of techniques and methods for figuratively representing the conditions of a problem, allowing either immediately see a solution or get a hint for finding it "[6]. Domestic researchers have made a significant contribution to the development of methods of cognitive graphics and visualization of information. These include D.A. Pospelov [7], A.A. Zenkin [6], A.A. Bashlykov [8], M.N. Burdaev [9], A.E. Yankovskaya [10], V.G. Grishin [11], A.Yu. Zinoviev [12], Yu.V. Novoselov [13], and others. Among foreign researchers, the works of D.E. Kieras [14], T. Höllt [15], and F. Fischer [16] are to be noted.

The proposed method is universal and can be used not only to control the noisiness of electronic signals but also to monitor failures of spacecraft orientation sensors, the state of the equipment of the GS CIS. The mentioned image-building algorithms can be used in other applied fields, for example, for monitoring and diagnosing power plants, or for determining the severity of a patient's disease in medicine.

\section{The formation of a cognitive image of electronic signals based on the polar scan}

Table 1 presents the formulas that can be used to create polar scans of electronic signals. Moreover, formula (1) ("star") was proposed by Grishin [11], and the authors proposed formulas (2-5) in this paper.

The following notation is used in the table: $x=\left(x_{1}, \ldots, x_{n}\right)-$ a vector elements of which are informative signal parameters. Besides, each $i$-point of the contour has coordinates $(\varphi, \rho(\varphi))$, where $0 \leq \varphi<360$.

Table 1 - Methods for representing polar scans

\begin{tabular}{|c|c|c|c|}
\hline No. & $\begin{array}{c}\text { Cognitive image of a sinus- } \\
\text { oid }\end{array}$ & $\begin{array}{l}\text { Formula } \\
\text { Number }\end{array}$ \\
\hline 1 & $\rho(\varphi)=\sum_{k=1}^{n} x_{k} \sin (k \varphi)$ & \\
\hline & $\rho(\varphi)=\sum_{k=1}^{n} x_{k}\left(\cos (k \varphi)+\sin ^{k}(k \varphi)\right)$ & \\
\hline & $\rho(\varphi)=\sum_{k=1}^{n} x_{k}(1+k(\cos (k \varphi)+\sin (k \varphi)))$
\end{tabular}




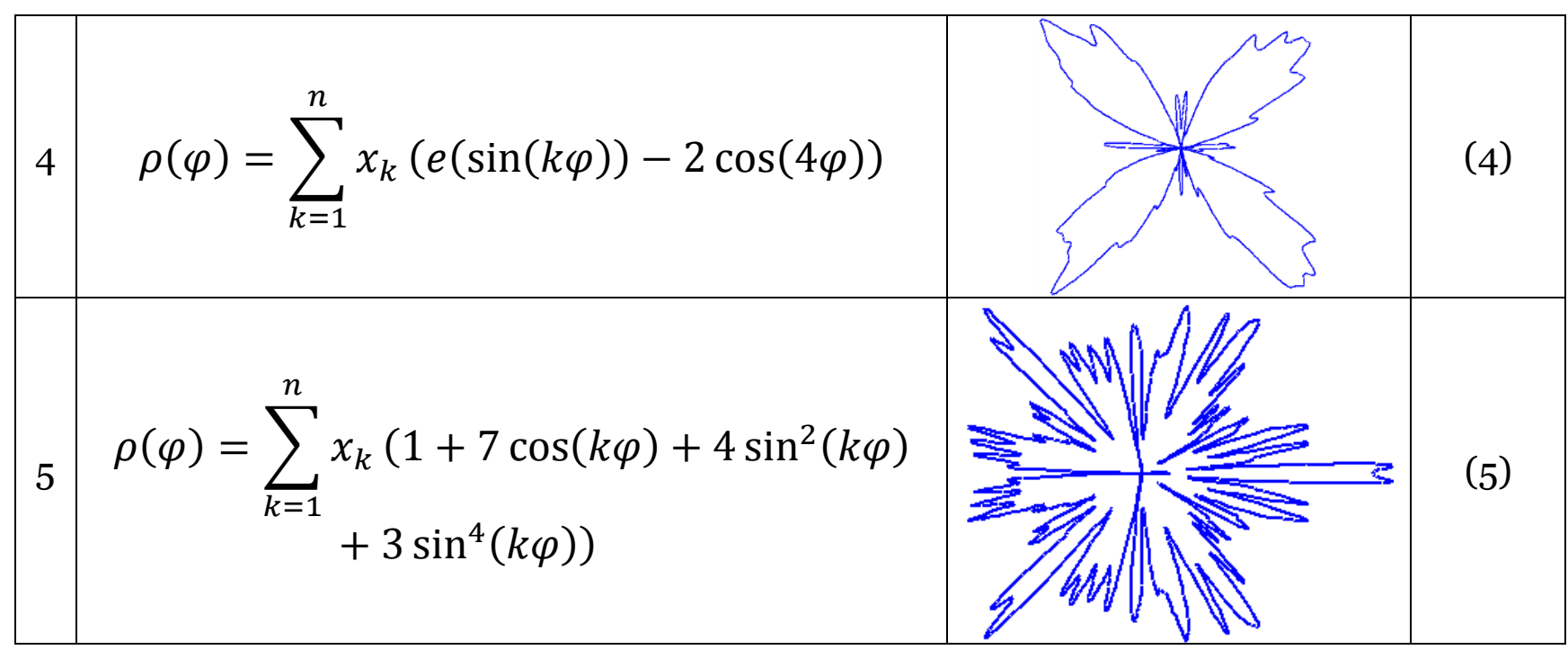

It is noted that the more complex the polar scan formula, the less visual is the image, and the more distinctive features are hidden. It is proposed to use the most simple and, at the same time, convenient for perception, the polar scan of the "star" type for the formation of cognitive-graphic images. Listed below are the informative signal parameters used in constructing the contour image [17-20] (table 2).

Table 2 - Informative signal parameters

\begin{tabular}{|c|c|c|}
\hline No. & Name & Formula \\
\hline 1 & Average power for a discrete signal & $\begin{array}{l}P_{\mathrm{cp}}=\frac{1}{N} \sum_{n=0}^{N-1} s_{n}^{2} \text { where } N \text { is the number of } \\
\text { discrete samples of the signal, } s_{n} \text { are the val- } \\
\text { ues of discrete samples of the signal }\end{array}$ \\
\hline 2 & $\begin{array}{l}\text { The quadratic mean value for the signal } \\
\text { sampling period }\end{array}$ & $\sqrt{\frac{1}{N}} \sum_{n=0}^{N-1} s_{n}^{2}$ \\
\hline 3 & $\begin{array}{l}\text { The average value for the signal sampling } \\
\text { period }\end{array}$ & $M[S]=\frac{1}{N} \sum_{n=0}^{N-1} S_{n}$ \\
\hline 4 & $\begin{array}{l}\text { The quadratic mean value for the signal } \\
\text { sampling period }\end{array}$ & $\frac{1}{N} \sum_{n=0}^{N-1}\left|s_{n}\right|$ \\
\hline 5 & $\begin{array}{l}\text { The sum of the amplitude-frequency } \\
\text { characteristics (AFC) }\end{array}$ & $\begin{array}{l}\sum_{m=0}^{N-1} \sqrt{A_{m}^{2}+B_{m}^{2}}, \text { where } \\
A_{m}=\sum_{n=0}^{N-1} s_{n} \cos (2 \pi n m / N) \\
B_{m}=\sum_{n=0}^{N-1} s_{n} \sin (2 \pi n m / N)\end{array}$ \\
\hline 6 & Discrete signal energy & $E=\sum_{n=0} s_{n}^{2}$ \\
\hline 7 & Discrete signal duration & $\begin{array}{l}T=N \cdot \Delta t \text { where } N \text { is the number of dis- } \\
\text { crete samples, } \Delta t \text { is the sampling period }\end{array}$ \\
\hline 8 & $\begin{array}{l}\text { Efficient signal spectrum width is the fre- } \\
\text { quency band within which the primary } \\
\text { signal energy is concentrated }\end{array}$ & $\begin{array}{l}\Delta \omega_{\ni \phi}=\frac{1}{F_{\max }} \sum_{n=0}^{N-1} F(\omega), \text { where } F(\omega) \text { is the } \\
\text { one-way power spectrum } \\
F_{\max }=\max (F(\omega)) .\end{array}$ \\
\hline
\end{tabular}




\begin{tabular}{|c|c|c|}
\hline 9 & $\begin{array}{l}\text { The signal base is the product of the sig- } \\
\text { nal duration by the effective width of its } \\
\text { spectrum }\end{array}$ & $B=\Delta \omega_{\ni \phi} T$ \\
\hline 10 & Signal/Noise ratio & $\begin{array}{l}\frac{M[S]}{\sigma[S]}, \text { where } M[S] \text { is the mean value of the } \\
\text { signal, } \sigma[S] \text { is the standard deviation of the } \\
\text { signal }\end{array}$ \\
\hline 11 & Dynamic Range & $\begin{array}{l}D=10 \lg \frac{P_{\max }}{P_{\min }} \text {, where } P_{\max } \text { is the maximum } \\
\text { instantaneous signal power, } P_{\min } \text { is the min- } \\
\text { imum instantaneous signal power }\end{array}$ \\
\hline 12 & The amount of information transmitted & $V=\Delta \omega_{\ni \phi} T D$ \\
\hline 13 & Dispersion & $D[S]=\frac{1}{N} \sum_{n=0}^{N-1}\left(s_{n}-M[S]\right)$ \\
\hline 14 & Standard deviation & $\sigma=\sqrt{D[S]}$ \\
\hline 15 & Signal rate & $\|s\|=\sqrt{\frac{1}{N}} \sum_{n=0}^{N-1} s_{n}^{2}$ \\
\hline 16 & $\begin{array}{l}\text { The minimum value of the signal in the } \\
\text { sampling period }\end{array}$ & $s_{\min }$ \\
\hline 17 & $\begin{array}{l}\text { The maximum value of the signal in the } \\
\text { sampling period }\end{array}$ & $s_{\max }$ \\
\hline 18 & Signal span & $s_{\max }-s_{\min }$ \\
\hline 19 & Extreme deviation from the mean value & $\max \left|s_{n}-M[S]\right|$ \\
\hline 20 & $\begin{array}{l}\text { The minimum value of the real part of the } \\
\text { spectrum }\end{array}$ & $\min \left(A_{m}\right)$ \\
\hline 21 & $\begin{array}{l}\text { The maximum value of the real part of } \\
\text { the spectrum }\end{array}$ & $\max \left(A_{m}\right)$ \\
\hline 22 & $\begin{array}{l}\text { Difference between the maximum and } \\
\text { minimum values of the real part of the } \\
\text { spectrum }\end{array}$ & $\max \left(A_{m}\right)-\min \left(A_{m}\right)$ \\
\hline 23 & $\begin{array}{l}\text { Sum of values of the real part of the spec- } \\
\text { trum }\end{array}$ & $\sum_{m=0}^{N-1} A_{m}$ \\
\hline 24 & $\begin{array}{l}\text { The minimum value of the imaginary part } \\
\text { of the spectrum }\end{array}$ & $\min \left(B_{m}\right)$ \\
\hline 25 & $\begin{array}{l}\text { The maximum value of the imaginary } \\
\text { part of the spectrum }\end{array}$ & $\max \left(B_{m}\right)$ \\
\hline 26 & $\begin{array}{l}\text { Difference between the maximum and } \\
\text { minimum values of the imaginary part of } \\
\text { the spectrum }\end{array}$ & $\max \left(B_{m}\right)-\min \left(B_{m}\right)$ \\
\hline 27 & $\begin{array}{l}\text { The sum of the imaginary part of the } \\
\text { spectrum }\end{array}$ & $\sum_{m=0}^{N-1} B_{m}$ \\
\hline
\end{tabular}




\begin{tabular}{|l|l|l|}
\hline 28 & FRC minimum value & $\min \left(R_{m}\right)$, where $R_{m}=\sqrt{A_{m}^{2}+B_{m}^{2}}$ \\
\hline 29 & FRC maximum value & $\max \left(R_{m}\right)$ \\
\hline 30 & $\begin{array}{l}\text { The difference between the maximum } \\
\text { and minimum values of the frequency re- } \\
\text { sponse curve }\end{array}$ & $\max \left(R_{m}\right)-\min \left(R_{m}\right)$ \\
\hline 31 & Correlation interval & $\tau_{K}=\frac{\pi}{2 \Delta \omega_{\ni \phi}}$ \\
\hline 32 & $\begin{array}{l}\text { The minimum value of the phase- } \\
\text { frequency characteristic (PFC) }\end{array}$ & $\min \left(\varphi_{m}\right)$, where $\varphi_{m}=\operatorname{arctg}\left(\frac{-B_{m}}{A_{m}}\right)$ \\
\hline 33 & PFC maximum value & $\max \left(\varphi_{m}\right)$ \\
\hline 34 & $\begin{array}{l}\text { The difference between the maximum } \\
\text { and minimum values of the PFC }\end{array}$ & $\max \left(\varphi_{m}\right)-\min \left(\varphi_{m}\right)$ \\
\hline 35 & PFC total & $\begin{array}{l}N-1 \\
m=0\end{array}$ \\
\hline 36 & Current spectrum over time $T$ & $S_{T}=\sum_{m=0}^{N-1} R_{m} e^{\varphi_{m}}$ \\
\hline 37 & Signal to noise ratio in decibels (dB) & $Q=10 \lg \frac{P_{\mathrm{cp}}}{D[S]}$, where $P_{\mathrm{cp}}$ is the average in- \\
\hline 38 & Maximum raw bandwidth & $\mathrm{C}=\Delta \omega_{\ni \phi} \log \sin _{2}(1+Q)$ \\
\hline 39 & Probability integral & $\Phi(s)=\sum_{n=0}^{N-1} e^{-s_{n}^{2} / 2}$ \\
\hline 40 & RMS noise voltage & $\sqrt{F_{\max } \Delta \omega_{\ni \phi}}$ \\
\hline
\end{tabular}

The operator receives the opportunity to independently form a cognitive image by choosing the composition of the vector $x$ from the specified parameters.

The order of the coordinates of the vector $x=\left(x_{1}, \ldots, x_{n}\right)$ is not random: the first coordinates of the vector determine the nature of the low-frequency components of the image (orientation, symmetry, etc.), and the last coordinates determine the high-frequency (local features) [11]. Rearrangements of coordinates in a vector of informative parameters $x=$ $\left(x_{1}, \ldots, x_{n}\right)$ can significantly change the ability to detect differences between cognitive images for mixed signals. Since the representation of more than twenty to thirty different ones in one circuit $x_{k}$ is inefficient, the dimension of the attribute space should be reduced. The ranking of features in descending order of informativity increases the selectivity of the polar sweep of the curve with the spectrum specified by the vector $x$. The ranking task is formulated as a search for a combination and order of features that correctly recognize the situation (electronic signal), allowing to get the most expressive cognitive images of discrete signals. It is proposed to form and rank a set of significant features by using the Add and Del algorithms $[21,22]$. The application of these algorithms at each step requires recognition of the type of signal and assessment of the quality of recognition. The recognition problem is formulated as follows. 
To build a recognition function $F(\omega), F(\omega)=\left(F_{1}(\omega), \ldots, F_{k}(\omega), \ldots, F_{m}(\omega)\right)$ the output of which determines the class $\Omega_{k}$ of an arbitrary image $\omega$ represented by a feature tor $\left(x_{1}(\omega), \ldots, x_{n}(\omega)\right)$.

$$
F_{k}(\omega)=\left\{\begin{array}{lc}
1, & \text { если } \omega \in \Omega_{k} \\
0, & \text { если } \omega \notin \Omega_{k} \\
\Delta, & \text { если неизвестно } \omega \in \Omega_{k} \text { или } \omega \notin \Omega_{k}
\end{array} .\right.
$$

It is proposed to use the Euclidean-Mahalanobis metric [23, 24], which describes the distribution of classes quite well to measure the distances between the sample and the class. As a measure of the quality of classification, the F-measure [25] was chosen: $F=2 \frac{P R}{P+R}$, where $P$ is the recognition accuracy, $R$ is the completeness of recognition.

Let $P_{u}=\left\{P_{1}, \ldots, P_{u}, \ldots, P_{s}\right\}-$ is a training sample of $s$ signal types $(s=15) . P_{u}$ - is a set of precedents of a class with a number $u$. The sample structure is presented in table 3 .

Table 3 - The structure of the training sample

\begin{tabular}{|c|c|c|c|c|c|c|}
\hline \multirow{2}{*}{ Objects } & \multicolumn{5}{|c|}{ Characteristics } & \multirow{2}{*}{$\begin{array}{c}\text { Groups of objects, } \\
\text { name }\end{array}$} \\
\hline & $x_{1}$ & $x_{2}$ & $x_{3}$ & $\ldots$ & $x_{40}$ & \\
\hline 1 & $x_{1}^{1,1}$ & $x_{2}^{1,1}$ & $x_{3}^{1,1}$ & $\cdots$ & $x_{40}^{1,1}$ & \multirow{3}{*}{$\begin{array}{c}P_{1} \\
\text { amplitude-modulated } \\
\text { signal }\end{array}$} \\
\hline 2 & $x_{1}^{1,2}$ & $x_{2}^{1,2}$ & $x_{3}^{1,2}$ & $\cdots$ & $x_{40}^{1,2}$ & \\
\hline 3 & $x_{1}^{1,3}$ & $x_{2}^{1,3}$ & $x_{3}^{1,3}$ & $\cdots$ & $x_{40}^{1,3}$ & \\
\hline 4 & $x_{1}^{2,1}$ & $x_{2}^{2,1}$ & $x_{3}^{2,1}$ & $\cdots$ & $x_{40}^{2,1}$ & \multirow{3}{*}{$\begin{array}{c}P_{2}, \\
\text { Dirichlet Function }\end{array}$} \\
\hline$\cdots$ & \multicolumn{5}{|c|}{$\cdots$} & \\
\hline 7 & $x_{1}^{2,4}$ & $x_{2}^{2,4}$ & $x_{3}^{2,4}$ & $\cdots$ & $x_{40}^{2,4}$ & \\
\hline 8 & $x_{1}^{3,1}$ & $x_{2}^{3,1}$ & $x_{3}^{3,1}$ & $\cdots$ & $x_{40}^{3,1}$ & \multirow{3}{*}{$\begin{array}{c}P_{3}, \\
\text { Dirichlet Function } \\
\text { with noise }\end{array}$} \\
\hline 9 & $x_{1}^{3,2}$ & $x_{2}^{3,2}$ & $x_{3}^{3,2}$ & $\cdots$ & $x_{40}^{3,2}$ & \\
\hline 10 & $x_{1}^{3,3}$ & $x_{2}^{3,3}$ & $x_{3}^{3,3}$ & $\cdots$ & $x_{40}^{3,3}$ & \\
\hline 11 & $x_{1}^{4,1}$ & $x_{2}^{4,1}$ & $x_{3}^{4,1}$ & $\cdots$ & $\begin{array}{l}x_{40}^{4,1} \\
\end{array}$ & \multirow{2}{*}{$\begin{array}{c}P_{4}, \\
\text { Gauss's impulse }\end{array}$} \\
\hline 12 & $x_{1}^{4,2}$ & $x_{2}^{4,2}$ & $x_{3}^{4,2}$ & $\cdots$ & $\begin{array}{l}x_{40}^{4,2} \\
\end{array}$ & \\
\hline$\cdots$ & \multicolumn{5}{|c|}{$\cdots$} & $\ldots$ \\
\hline 49 & $x_{1}^{15,1}$ & $x_{2}^{15,1}$ & $x_{3}^{15,1}$ & $\cdots$ & $x_{40}^{15,1}$ & \multirow{2}{*}{$\begin{array}{c}P_{15}, \\
\text { triangular impulse } \\
\text { with noise }\end{array}$} \\
\hline 50 & $x_{1}^{15,2}$ & $x_{2}^{15,2}$ & $x_{3}^{15,2}$ & $\cdots$ & $x_{40}^{15,2}$ & \\
\hline
\end{tabular}

Algorithm 1. (Isolation and normalization of informative parameters. Formation of a training sample).

Given: a set of typical signals presented in a discrete form with a step of 0.2. Each type of signal in table 3 corresponds to several precedents (from one to six).

Result: a normalized training sample of 40 informative parameters.

Informative signal parameters for each training sample are calculated using the formulas (140, table 2).

1. The obtained characteristics are normalized: for each informative parameter according to the formula $\bar{x}_{k}^{u, b}=\frac{x_{k}^{u, b}-x_{k \_ \text {min }}}{x_{k \_ \text {max }}-x_{k_{-} \min }}$, where $x_{k}^{u, b}$ - the value $k$ of the ith informative param- 
eter of the signal with the number $b$ in the subsample with the number $u, x_{k \_ \text {min }}$ - the minimum value $k$ of the ith informative parameter, $x_{k_{-} \max }$ - the maximum value $k$ of the ith informative parameter.

2. The mathematical expectations of normalized parameters are calculated for all types of signals $\left(M_{k}^{u}=\frac{1}{N} \sum_{b=1}^{N} \bar{x}_{k}^{u, b}\right.$ where $N$ - is the number of objects in the subsample $\left.P_{u}\right)$.

3. For each type of signal, the inverse covariance matrix is calculated (the elements of the covariance matrices are calculated as follows:

$\left.c_{u i j}=\frac{1}{N-1} \sum_{b=1}^{N}\left(\bar{x}_{i}^{u, b}-M_{i}^{u}\right)\left(\bar{x}_{j}^{u, b}-M_{j}^{u}\right), i, j=1, \ldots, 40\right)$.

Table 4 presents the Euclidean distances between the images following the adopted feature space.

Table 4 - Euclidean distances between the reference signals

\begin{tabular}{|c|c|c|c|c|c|c|}
\hline № & $\begin{array}{c}\text { Название эталонного } \\
\text { сигнала }\end{array}$ & 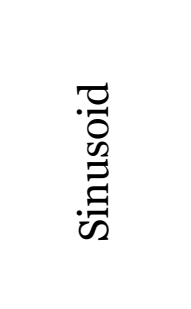 & 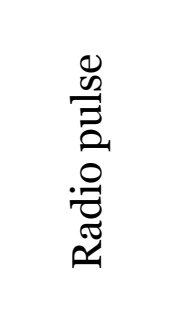 & 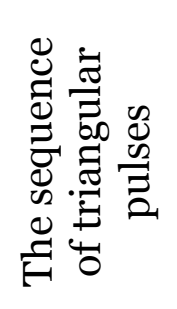 & 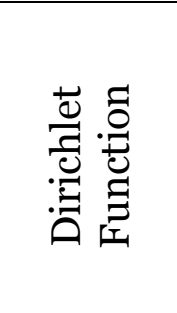 & 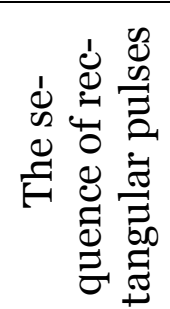 \\
\hline 1 & Sinusoid & $\mathrm{O}$ & 1.06234 & 0.98495 & 1.26333 & 0.92407 \\
\hline 2 & Radio pulse & 1.06234 & 0 & 1.32460 & 1.54736 & 1.32216 \\
\hline 3 & $\begin{array}{l}\text { The sequence of trian- } \\
\text { gular pulses }\end{array}$ & 0.98495 & 1.32460 & $\mathrm{O}$ & 0.80873 & 0.78621 \\
\hline 4 & Dirichlet Function & 1.26333 & 1.54736 & 0.80873 & $\mathrm{O}$ & 0.80384 \\
\hline 5 & $\begin{array}{l}\text { The sequence of rec- } \\
\text { tangular pulses }\end{array}$ & 0.92407 & 1.32216 & 0.78621 & 0.80384 & $\mathrm{O}$ \\
\hline
\end{tabular}

Table 5 - Euclidean-Mahalanobis distances between the reference signals

\begin{tabular}{|c|c|c|c|c|c|c|}
\hline № & Reference signal name & 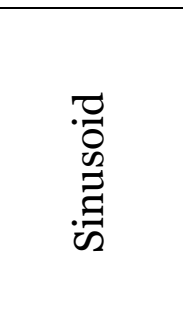 & 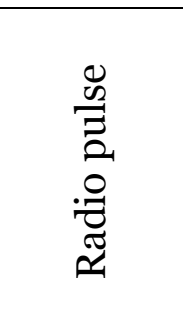 & 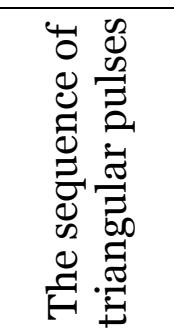 & 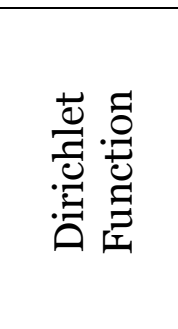 & 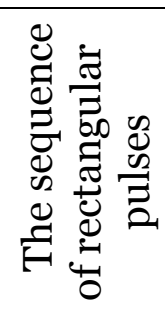 \\
\hline 1 & Sinusoid & $\mathrm{O}$ & 1.12659 & 0.96096 & 1.58214 & 0.84224 \\
\hline 2 & Radio pulse & 1.12659 & 0 & 1.75327 & 2.40006 & 1.72658 \\
\hline 3 & $\begin{array}{l}\text { The sequence of trian- } \\
\text { gular pulses }\end{array}$ & 0.96096 & 1.75327 & $\mathrm{O}$ & 0.65090 & 0.13895 \\
\hline 4 & Dirichlet Function & 1.58214 & 2.40006 & 0.65090 & $\mathrm{O}$ & 0.62974 \\
\hline 5 & $\begin{array}{l}\text { The sequence of rec- } \\
\text { tangular pulses }\end{array}$ & 0.84224 & 1.72658 & 0.13895 & 0.62974 & $\mathrm{O}$ \\
\hline
\end{tabular}

\section{Optimization of a set of informative features}

Consider the combined use of Del and Add methods. First, the informative features are sorted using the Add method. Then, the obtained set of attributes is inverted, ranked in information content descending order. Next, a repeated ranking of the characteristics is performed, but according to the Del method. We get a vector of signs, ranked by increasing information content. The inverse of the resulting feature vector is performed.

Consider using the Add and Del methods in a different order. First, the informative features are sorted using the Del method. Then, an inversion of the obtained set of features, ranked by 
increasing information content, is performed. Next, the characteristics are ranked using the Add method. As a result, we obtain a vector of signs, ranked in descending order of informativeness.

Table 6 presents the results of the selection of the most informative images by combined methods using procedures $\operatorname{Add}\left(\operatorname{Del}\left(x_{1}, \ldots, x_{n}\right)\right)$ and $\operatorname{Del}\left(\operatorname{Add}\left(x_{1}, \ldots, x_{n}\right)\right)$. The distances of Euclidean and Euclidean-Mahalanobis from the considered signal to its standard are given. Table 6 - Cognitive graphic images of signals

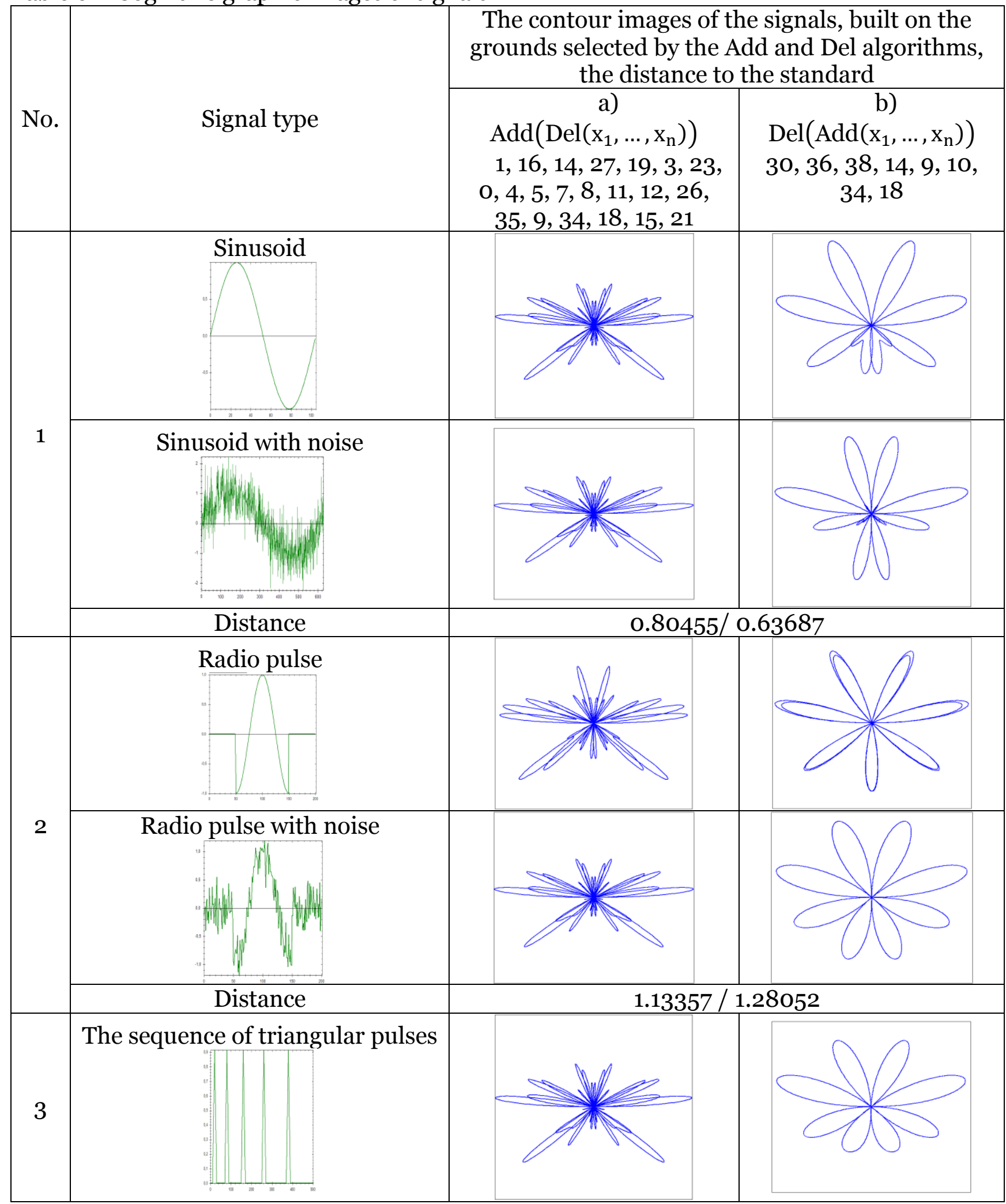




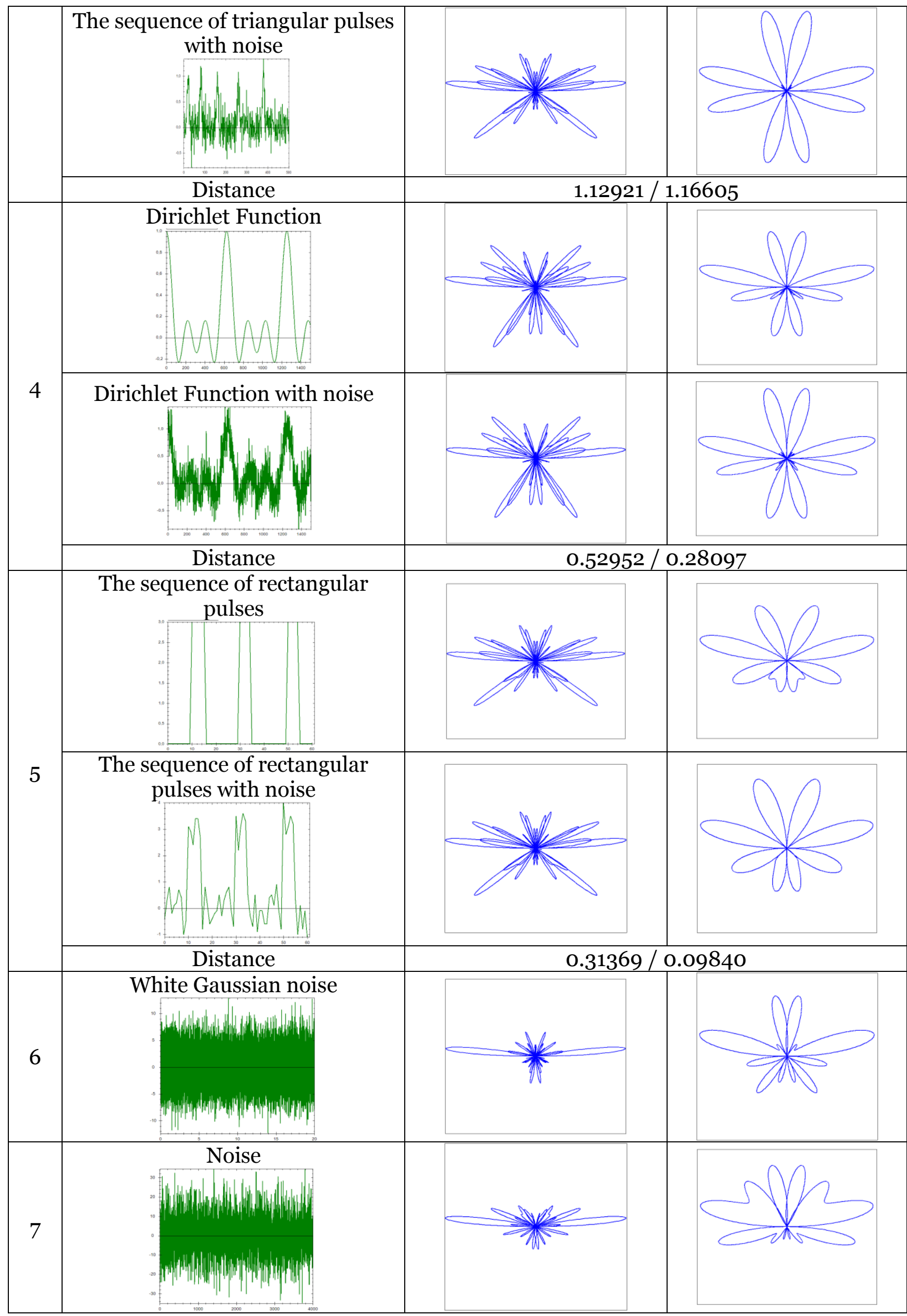


The most distinguishable images for individual types of signals are formed by automatic permutation and selection of parameters based on a combination of Del and Add methods (option b). Therefore, in the future, when constructing polar scans, we take a sequence of features following option b) (table 6) as a basis. Algorithm 2 and Algorithm 3 describe two sequential procedures $\operatorname{Del}\left(\operatorname{Add}\left(x_{1}, \ldots, x_{n}\right)\right)$ for ordering features in support of this option.

\section{Algorithm 2. (Feature Ranking: Add Algorithm)}

Given: InfParamNumbers - vector numbers of informative signs.

AddSortParams $=\varnothing$.

Result: AddSortParams is - a vector of numbers of informative parameters ranked by increasing information content.

1. If the InfParamNumbers informative parameter number vector is empty, go to step 7 .

2. The AddSortParams vector is increased by one element to the right, which is assigned the number of the first informative parameter from the InfParamNumbers vector.

3. The recognition of the AddSortParams sequence is performed.

4. The F-measure [25] of recognition quality, the maximum F-measure and the number of the corresponding informative parameter are calculated.

5. The last element of the AddSortParams vector is assigned the number of the informative parameter at which the F-measure is maximum.

6 . The number of the informative parameter on which the AddSortParams sequence received the maximum F-measure is removed from the InfParamNumbers vector. Go to step 1.

7. Reverse order of the ranked feature vector AddSortParams.

8. The end.

\section{Algorithm 3. (Feature Ranking: Del Algorithm)}

Given: AddSortParams; DelAddSortParams is - a set of numbers of informative features sorted by the Add and Del methods, respectively; DelAddSortParams $=\varnothing$.

Result: DelAddSortParams - a set of numbers of informative parameters ranked in descending order of informativeness.

1. If the AddSortParams informative parameter number vector is empty, go to step 9.

2. Let $i=o$ be the number of the checked informative parameter, and length be the length of the AddSortParams vector.

3. Recognition of the signal described by the tmpList vector, which includes all the features from the AddSortParams vector, except for the $i$-one.

4. The F-measure of recognition quality is calculated.

5. $i$ increases by one. If $i \leq$ length, go to step 3; otherwise, go to step 6 .

6 . The maximum F-measure and the number of the corresponding informative parameter are calculated.

7. The vector DelAddSortParams is increased by one element to the right, which is assigned the number of the informative parameter at which the F-measure is maximum.

8. The number of the informative parameter on which the tmpList sequence received the maximum F-measure is deleted From the AddSortParams vector. Length decreases by one. Go to step 1.

9. Reverse order of the ranked feature vector DelAddSortParams.

10. The end.

The result of the algorithms is presented in table 7.

Table 7 - Signs Ranked by Procedure $\operatorname{Del}\left(\operatorname{Add}\left(x_{1}, \ldots, x_{n}\right)\right)$

\begin{tabular}{|c|c|l|}
\hline Priority & Number & \multicolumn{1}{|c|}{ Name } \\
\hline 1 & $\mathbf{3 0}$ & Correlation interval \\
\hline 2 & $\mathbf{3 6}$ & Signal to noise ratio in decibels \\
\hline 3 & $\mathbf{3 8}$ & Probability integral \\
\hline 4 & $\mathbf{1 4}$ & Signal rate \\
\hline
\end{tabular}




\begin{tabular}{|c|c|c|}
\hline 5 & 9 & Signal/Noise ratio \\
\hline 6 & 10 & Dynamic Range \\
\hline 7 & 34 & PFC total \\
\hline 8 & 18 & Extreme deviation from the mean value \\
\hline 9 & 13 & Standard deviation \\
\hline 10 & 1 & The quadratic mean value for the signal sampling period \\
\hline 11 & 25 & $\begin{array}{l}\text { Difference between the maximum and minimum values of the imagi- } \\
\text { nary part of the spectrum }\end{array}$ \\
\hline 12 & 15 & The minimum value of the signal in the sampling period \\
\hline 13 & 17 & Signal span \\
\hline 14 & 16 & The maximum value of the signal in the sampling period \\
\hline 15 & 27 & FRC minimum value \\
\hline 16 & 3 & The average value for the signal sampling period \\
\hline 17 & 37 & Maximum raw bandwidth \\
\hline 18 & 39 & RMS noise voltage \\
\hline 19 & 22 & Sum of values of the real part of the spectrum \\
\hline 20 & 35 & Current spectrum \\
\hline 21 & 26 & The sum of the imaginary part of the spectrum \\
\hline 22 & 12 & Dispersion \\
\hline 23 & 11 & The amount of information transmitted \\
\hline 24 & 8 & The signal base \\
\hline 25 & 5 & Discrete signal energy \\
\hline 26 & 4 & The sum of the amplitude-frequency characteristics \\
\hline 27 & 24 & The maximum value of the imaginary part of the spectrum \\
\hline 28 & $\mathrm{O}$ & Average power for a discrete signal \\
\hline 29 & 19 & The minimum value of the real part of the spectrum \\
\hline 30 & 21 & $\begin{array}{l}\text { Difference between the maximum and minimum values of the real par } \\
\text { of the spectrum }\end{array}$ \\
\hline 31 & 6 & Discrete signal duration \\
\hline 32 & 29 & $\begin{array}{l}\text { The difference between the maximum and minimum values of the fre- } \\
\text { quency response curve }\end{array}$ \\
\hline 33 & 28 & FRC maximum value \\
\hline 34 & 7 & Efficient signal spectrum width \\
\hline 35 & 23 & The minimum value of the imaginary part of the spectrum \\
\hline 36 & 2 & The average value for the signal sampling period \\
\hline 37 & 20 & The maximum value of the real part of the spectrum \\
\hline 38 & 33 & The difference between the maximum and minimum values of the PFC \\
\hline 39 & 32 & Phase-frequency characteristic maximum value \\
\hline 40 & 31 & The minimum value of the phase-frequency characteristic \\
\hline
\end{tabular}

In table 7, a set of features is highlighted in italics, on which the procedure $\operatorname{Del}\left(\operatorname{Add}\left(x_{1}, \ldots, x_{n}\right)\right)$ receives the highest recognition quality. Let us compare signal recognition with metrics and visual recognition of polar scans.

Let us check the quality of signal recognition by the Euclidean metric within the feature space (table 8). 
Table 8 - Euclidean distance between reference signals and noisy signals

\begin{tabular}{|c|c|c|c|c|c|c|}
\hline No. & Reference signal name & 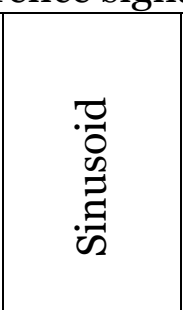 & 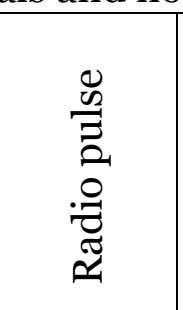 & 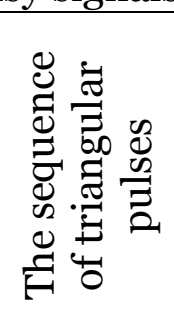 & 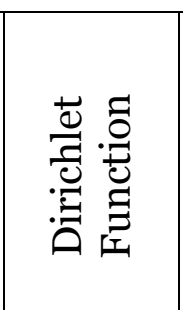 & 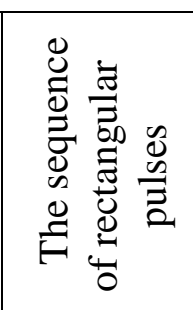 \\
\hline & & 1 & 2 & 3 & 4 & \\
\hline \multirow{3}{*}{1} & Low noise sinusoid & 0.64940 & 1.24301 & .18212 & .00343 & .06825 \\
\hline & Med & 70485 & 25022 & & 97683 & .06850 \\
\hline & & & 23801 & & 0.89017 & 1.04242 \\
\hline \multirow{3}{*}{2} & Lor & 35857 & 00181 & & 1.15041 & 0.85729 \\
\hline & Mediur & 42052 & 03007 & 0.84535 & 12740 & .84390 \\
\hline & & 0.75130 & 1.13357 & 0.45124 & 85127 & 0.55152 \\
\hline \multirow{3}{*}{3} & $\begin{array}{c}\text { Low noise sequence of triangu } \\
\text { es }\end{array}$ & 0.99473 & 1.32935 & 0.03409 & 0.80886 & 0.42113 \\
\hline & $\begin{array}{r}\text { Medium noise sequ } \\
\text { puls }\end{array}$ & 1.08651 & 1.38019 & 0.22642 & 0.76408 & 0.47037 \\
\hline & $\begin{array}{r}\text { High noise sequ } \\
\text { pu }\end{array}$ & 0.96515 & 1.26618 & 1.12921 & 0.85537 & 1.04897 \\
\hline \multirow{3}{*}{4} & & 29310 & 1.56942 & .84335 & 0.05343 & 0.83612 \\
\hline & Medium & & 1.54370 & 0.87880 & 0.13766 & 0.86568 \\
\hline & & 1.14000 & 1.41096 & 1.020711 & 0.54339 & 0.984698 \\
\hline \multirow{3}{*}{5} & $\begin{array}{r}\text { Low noise sequenc } \\
\text { puls }\end{array}$ & 0.94846 & 1.33490 & 0.44178 & 0.79439 & 0.07093 \\
\hline & $\begin{array}{l}\text { Medium noise sequence of rectangular } \\
\text { pulses }\end{array}$ & 0.98077 & 1.31781 & 0.39168 & 0.76840 & 0.13974 \\
\hline & $\begin{array}{c}\text { High noise sequence of rectangular } \\
\text { pulses }\end{array}$ & 0.77987 & 1.20778 & 0.53626 & 0.82209 & 0.33712 \\
\hline
\end{tabular}

In table 8, the names of incorrectly recognized signals by the Euclidean metric are indicated in red. Green indicates the smallest distance from a noisy signal to one of the standards, indicating that the signal is correctly classified. The red color indicates the distance, indicating that the signal is classified incorrectly.

Thus, from table 8, it follows that the Euclidean distance is unstable to signal recognition in the presence of noise. Let us check the quality of signal recognition by the EuclideanMahalanobis metric. (table 9).

Table 9 - Recognition of radio signals by the Euclidean-Mahalanobis metric

\begin{tabular}{|c|c|c|c|c|c|}
\hline \multirow{2}{*}{ Noise baseline } & \multicolumn{5}{|c|}{ Same of the reference signal } \\
\cline { 2 - 6 } & Sinusoid & Radio pulse & $\begin{array}{c}\text { The sequence of } \\
\text { triangular pulses }\end{array}$ & $\begin{array}{l}\text { Dirichlet } \\
\text { Function }\end{array}$ & $\begin{array}{c}\text { The sequence of } \\
\text { rectangular pulses }\end{array}$ \\
\hline No noise & Sinusoid & Radio pulse & $\begin{array}{c}\text { The sequence of } \\
\text { triangular pulses }\end{array}$ & $\begin{array}{c}\text { Dirichlet } \\
\text { Function }\end{array}$ & $\begin{array}{c}\text { The sequence of } \\
\text { rectangular pulses }\end{array}$ \\
\hline Low noise level & $\begin{array}{c}\text { Sinusoid } \\
\text { with noise }\end{array}$ & $\begin{array}{c}\text { Radio pulse } \\
\text { with noise }\end{array}$ & $\begin{array}{c}\text { The sequence of } \\
\text { triangular pulses }\end{array}$ & $\begin{array}{c}\text { Dirichlet } \\
\text { Function }\end{array}$ & $\begin{array}{c}\text { The sequence of } \\
\text { rectangular pulses }\end{array}$ \\
\hline $\begin{array}{c}\text { Average noise } \\
\text { level }\end{array}$ & $\begin{array}{c}\text { Sinusoid } \\
\text { with noise }\end{array}$ & $\begin{array}{c}\text { Radio pulse } \\
\text { with noise }\end{array}$ & $\begin{array}{c}\text { The sequence of } \\
\text { triangular pulses }\end{array}$ & $\begin{array}{c}\text { Dirichlet } \\
\text { Function }\end{array}$ & $\begin{array}{c}\text { The sequence of } \\
\text { rectangular pulses }\end{array}$ \\
\hline
\end{tabular}




\begin{tabular}{|c|c|c|c|c|c|}
\hline & & & with noise & with noise \\
\hline $\begin{array}{c}\text { High noise } \\
\text { level }\end{array}$ & $\begin{array}{c}\text { Sinusoid } \\
\text { with noise }\end{array}$ & $\begin{array}{c}\text { Radio pulse } \\
\text { with noise }\end{array}$ & Sinusoid with noise & $\begin{array}{c}\text { Sinusoid } \\
\text { with noise }\end{array}$ & $\begin{array}{c}\text { The sequence of } \\
\text { rectangular pulses } \\
\text { with noise }\end{array}$ \\
\hline
\end{tabular}

Table 9 shows in red the incorrect results of signal classification by the EuclideanMahalanobis metric. The table shows that the Euclidean-Mahalanobis metric sometimes does not detect low and medium noise in a signal, for example, in a sequence of triangular pulses, the Dirichlet function, and a sequence of rectangular pulses. Signals with a high noise level are sometimes not recognized correctly, for example, as a noisy sinusoid. On the other hand, the positive quality of the Euclidean-Mahalanobis metric is noise immunity.

\section{Methods for improving the quality of visual recognition of polar scans}

To improve the quality of visual recognition, we add monochrome halftones to cognitive images. The formula $\lambda_{i}=\left[c \frac{\rho\left(\varphi_{i}\right)-\rho(\varphi)_{\min }}{\rho(\varphi)_{\max }-\rho(\varphi)_{\min }}\right]$ determines the brightness of a halftone, where $c=255$ is the maximum brightness value, $\rho(\varphi)_{\min }$ is the minimum value among all $\rho(\varphi)$, $\rho(\varphi)_{\min } \in\left\{\rho(0), \ldots, \rho\left(\varphi_{i}\right), \ldots, \rho(359)\right\}, \rho(\varphi)_{\max }$ is the maximum value among all $\rho(\varphi), \rho(\varphi)_{\max } \in\left\{\rho(0), \ldots, \rho\left(\varphi_{i}\right), \ldots, \rho(359)\right\}$. Table 10 shows the effect of the noise level on the cognitive images of the signals (polar scans), the Euclidean and EuclideanMahalanobis distances between the noisy signal and its standard.

Table 10 - Monochrome images of radio signals with different noise levels

\begin{tabular}{|c|c|c|c|c|}
\hline \multicolumn{5}{|c|}{ (2) } \\
\hline \multicolumn{5}{|l|}{ Signal graph } \\
\hline \multicolumn{5}{|l|}{$\begin{array}{l}\text { Signal cognitive } \\
\text { image }\end{array}$} \\
\hline Distance & $\mathrm{o}$ & $0.65024 / 0.42172$ & $0.72656 / 0.52790$ & $0.80455 / 0.63687$ \\
\hline \multicolumn{5}{|c|}{ 2) Radio pulse } \\
\hline \multicolumn{5}{|l|}{ Signal graph } \\
\hline \multicolumn{5}{|l|}{$\begin{array}{l}\text { Signal cognitive } \\
\text { image }\end{array}$} \\
\hline Distance & $\mathrm{o}$ & $1.00092 / 1.0018$ & $1.03008 / 1.05363$ & 1.13357 / 1.28052 \\
\hline
\end{tabular}




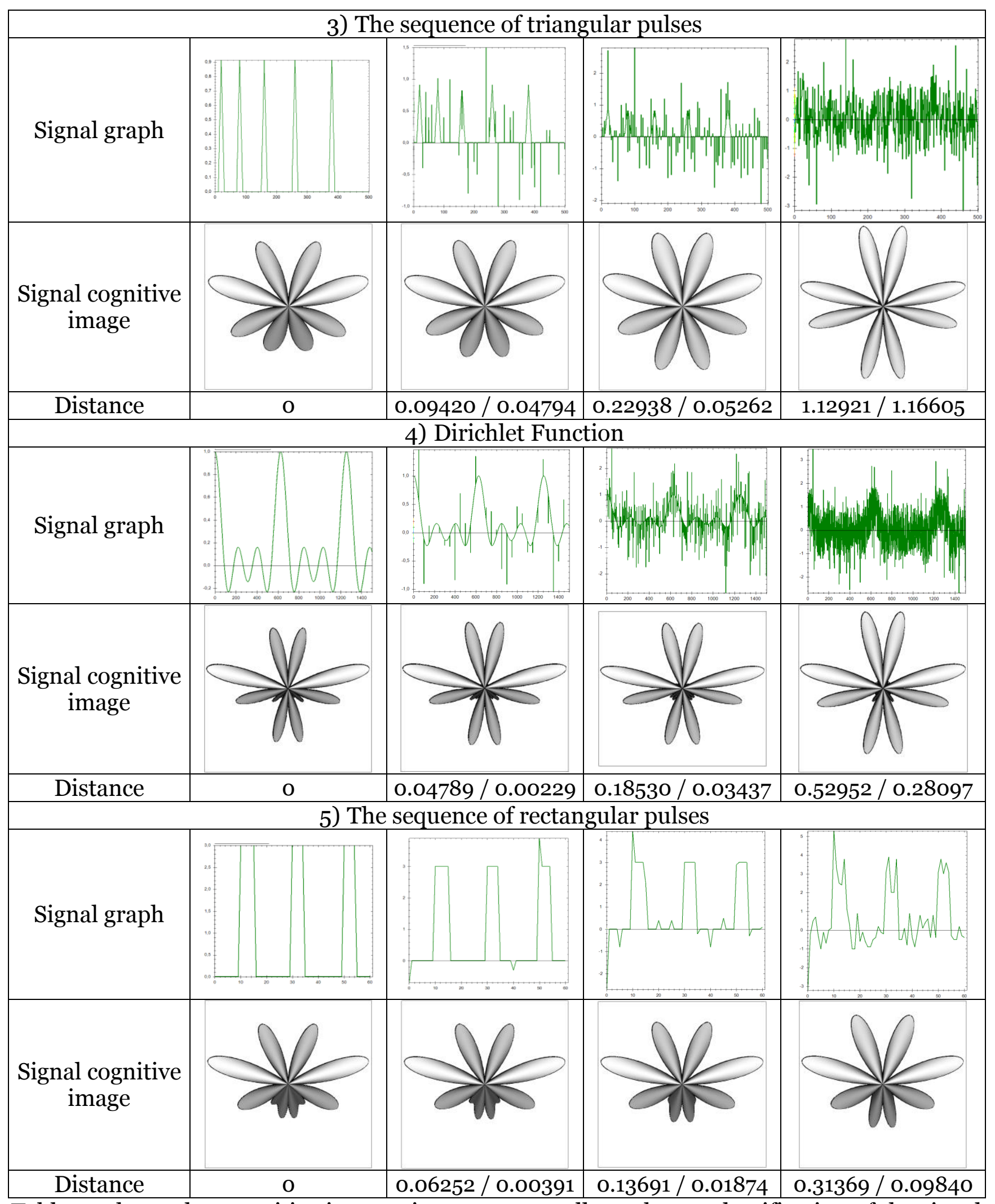

Table 10 shows that cognitive images, in most cases, allow a better classification of the signal than the Euclidean and Euclidean-Mahalanobis metrics. For example, a sinusoid with noise and the Dirichlet function with noise, classified by the Euclidean-Mahalanobis metric as the same signal (table 9), have similar, but visually distinguishable cognitive images. Namely: the lateral lower "rays" of a noisy sinusoid are noticeably shorter and darker than that of a noisy Dirichlet function. At the same time, at low and medium noise levels, the polar scans of the radio pulse and the sequence of triangular pulses differ very little, which complicates the visual recognition of the cognitive images of these signals. In other words, the recognition of 
electronic signals is qualitatively better when cognitive graphic images accompany the observation of numerical information and the classification of metrics.

To increase the clarity, let us introduce the operation of subtracting the signals defined over their signs: $\rho_{\text {разн }}(\varphi)=\sum_{k=1}^{n}\left(x_{k}-x_{k \ni}\right) \sin (k \varphi)$, where $x_{k \ni}-k$ is the $i$-reference feature of the signal. Let us perform the appropriate visualization $\rho_{\text {разн }}(\varphi)$ (table 11). Colorbrightness components are introduced into the contour representations of difference images to enhance perception, and the Euclidean-Mahalanobis distances to the reference signals in the adopted attribute space are given. The color calculation is performed according to the formula:

$$
(r, g, b)\left(\rho_{\text {разн }}(\varphi)\right)=\left\{\begin{array}{c}
\left(0, \eta\left(\rho_{\text {разн }}(\varphi)-\rho_{\text {разн }}(\varphi)_{\min }\right), c\right), \text { если } 0 \leq \rho_{\text {разн }}(\varphi)<\frac{\delta}{4} \\
\left(0, c, c-\eta\left(\rho_{\text {разн }}(\varphi)-\left(\rho_{\text {разн }}(\varphi)_{\min }+\frac{\delta}{4}\right)\right)\right), \text { если } \frac{\delta}{4} \leq \rho_{\text {разн }}(\varphi)<\frac{\delta}{2} \\
\left(\eta\left(\rho_{\text {разн }}(\varphi)-\left(\rho_{\text {разн }}(\varphi)_{\min }+\frac{\delta}{3}\right)\right), c, 0\right), \text { если } \frac{\delta}{2} \leq \rho_{\text {разн }}(\varphi)<\frac{3 \delta}{4} \\
\left(c, c-\eta\left(\rho_{\text {разн }}(\varphi)-\left(\rho_{\text {разн }}(\varphi)_{\min }+\frac{\delta}{2}\right)\right), 0\right), \text { если } \frac{3 \delta}{4} \leq \rho_{\text {разн }}(\varphi)<\delta \\
(c, 0,0), \text { если } \rho_{\text {разн }}(\varphi)=\rho_{\text {разн }}(\varphi)_{\text {max }}
\end{array},\right.
$$

where $(r, g, b)$ is the color code in RGB format, $\rho_{\text {разн }}(\varphi)_{\text {min }}$ is the minimum value among all $\rho_{\text {разн }}(\varphi), \rho_{\text {разн }}(\varphi)_{\max }$ is the maximum value among all $\rho_{\text {разн }}(\varphi), \delta=\rho_{\text {разн }}(\varphi)_{\max }-$ $\rho_{\text {разн }}(\varphi)_{\min }, \eta=\frac{4 c}{\delta}$.

Table 11 - The results of visualization of the difference of the investigated and reference signals

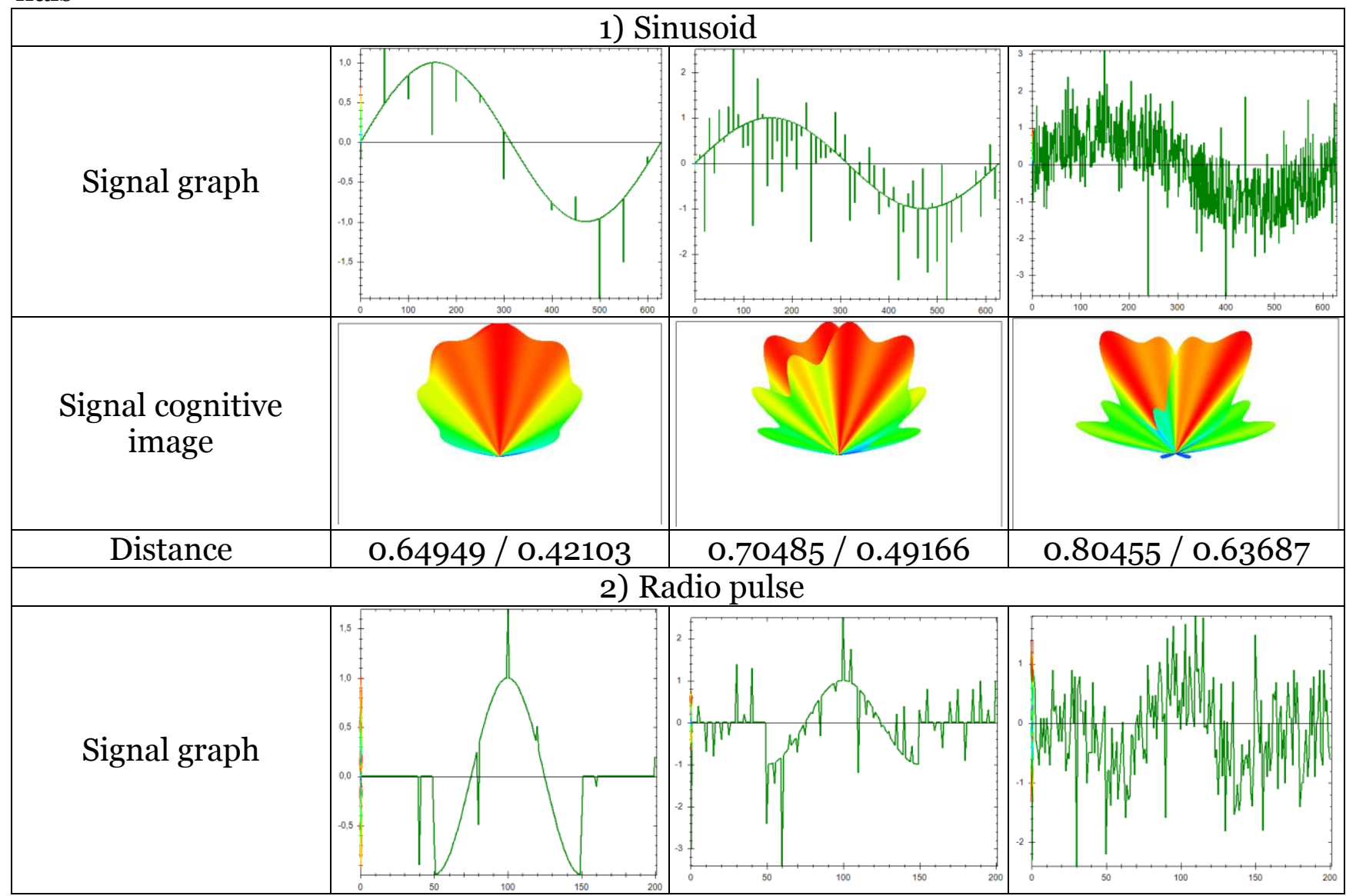




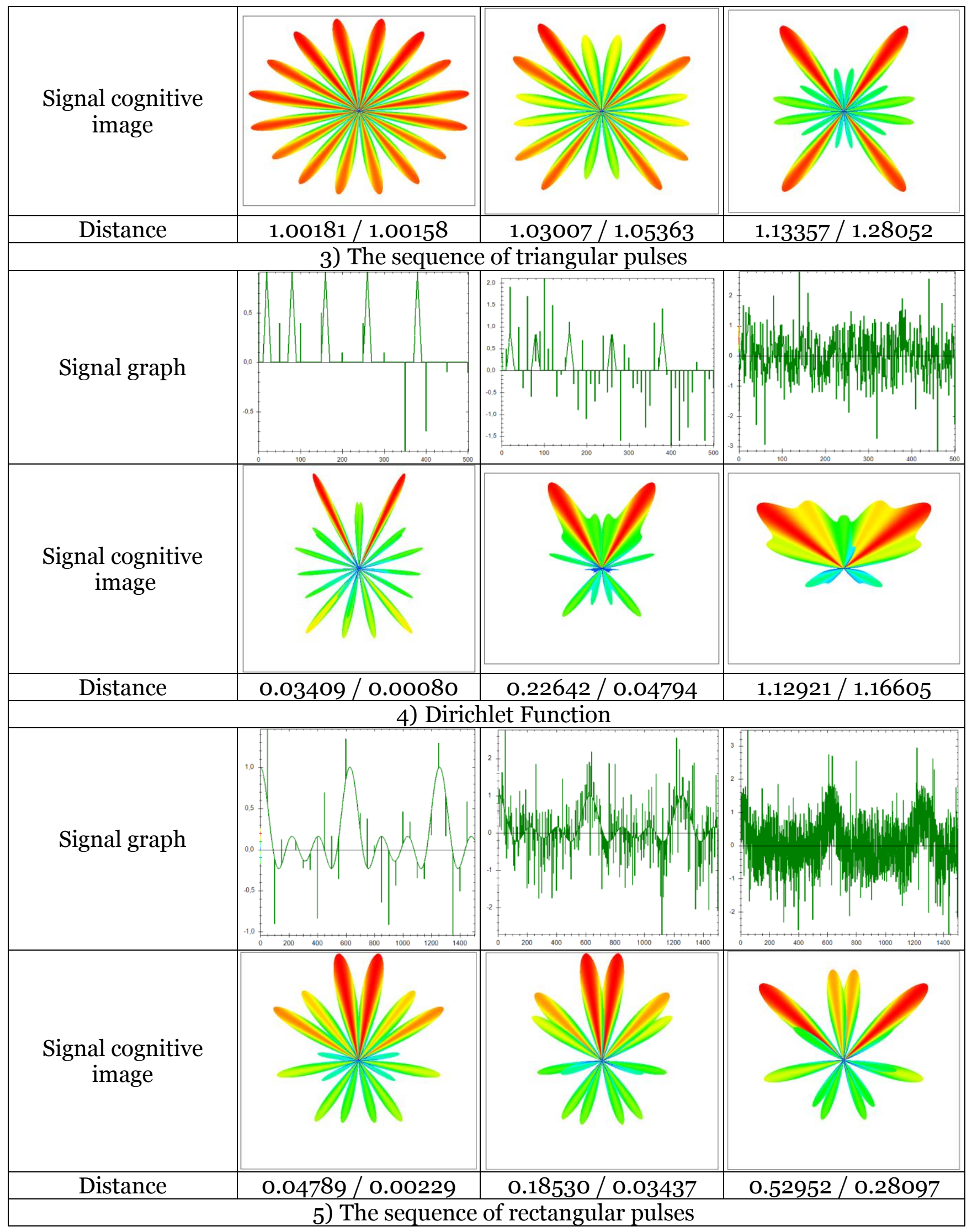




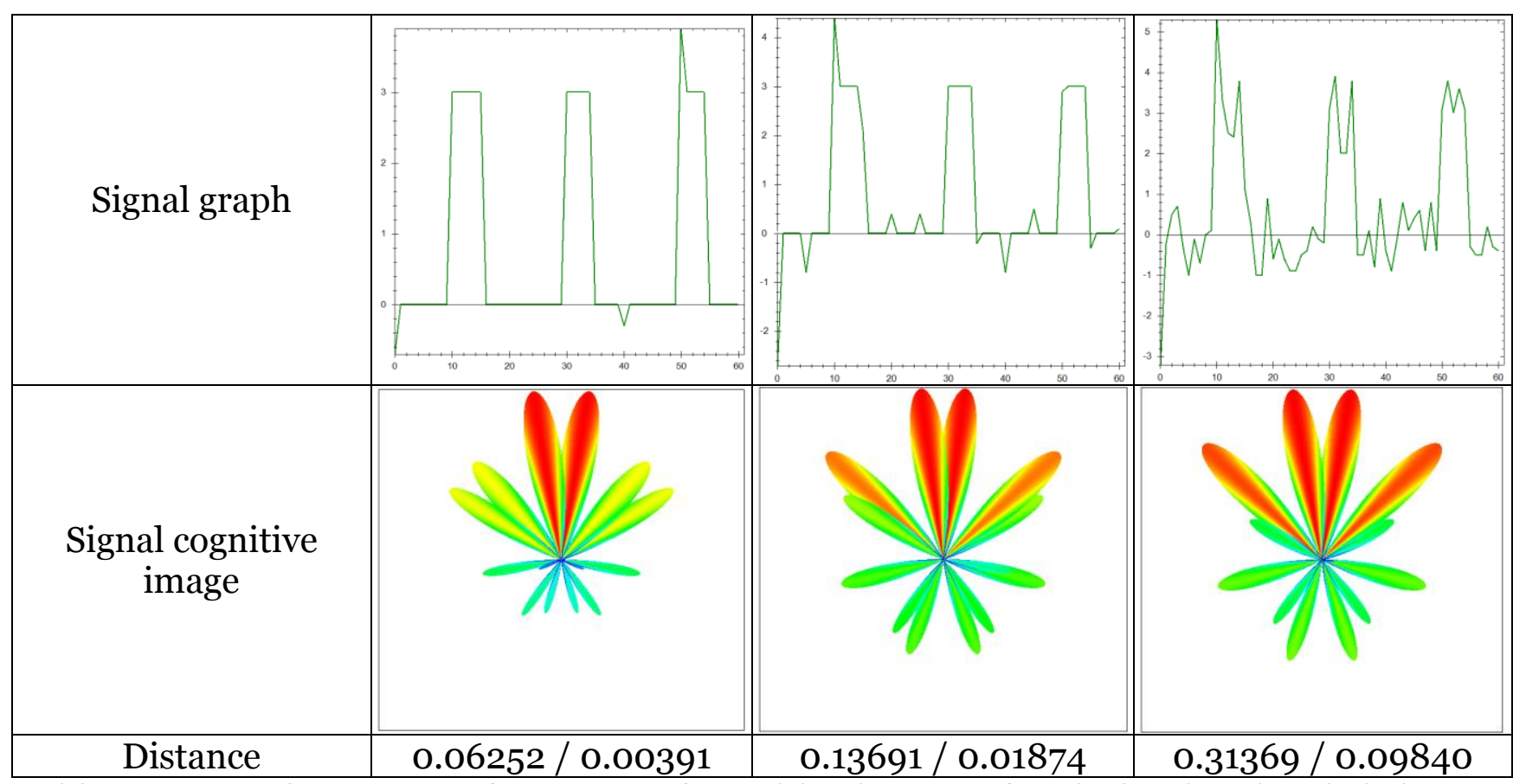

Tables 6, 10, and 11 present fragments of cognitive images of typical noisy signals being researched. The tables show the nature of the sensitivity of cognitive images to significant noise of signals evaluated using the entered distance. It is expressed in a change in the shape of polar scans (tables $6,10,11$ ), as well as in a change in the shape and color-brightness representations in difference cognitive images (table 11). Only the GS CIS operator who has undergone appropriate training can distinguish images. It is recommended that the interface provide both cognitive signal images and the results of their machine classification to achieve highquality signal recognition. Namely, as a result of experimental studies, it was shown that the type of signal is better to set using an automatic recognition program, which gives a recognition quality of $92.7 \%$. The operator needs to determine the noise level visually. Recommendations are given in the next section 4.

\section{Analysis and interpretation of cognitive images of sig- nals}

The operator of the GS CIS should visually determine the type of signal and its degree of noise by the presented cognitive image. As a recommendation for the operator, we indicate the following patterns that help the analysis, memorization, and interpretation of cognitive images.

\section{Signal Type Analysis}

Depending on the distances (tables $4,5,8$ ), the electronic signals built with the ranking of attributes performed have different cognitive images (tables $6,10,11$ ). It is especially noticeable in differenced color-brightness representations. All this allows the operator to distinguish (classify) them clearly.

\section{Noise Level Analysis}

From the analysis of tables $6,10,11$, first of all, it is clear that there is a correlation between the measured distances and the cognitive images of noisy signals:

- the greater the distance between the image of the signal and its standard, within the framework of the attribute space, the noisier it is;

- the more significant the difference in distances (interval) between noisy images of one class, the more they are distinguishable from each other.

Since the distance between the signals can be insignificant, and the corresponding images are poorly distinguishable, it is advisable, for the convenience of the operator, to divide the entire distance scale and, accordingly, the types of cognitive images into separate distinguishable subclasses, per the noise level: low noise, medium noise, high noise. From the analysis of the 
images, it is seen that during the transition from one subclass to another, the following general laws are observed: the shape, size and color gamut change significantly (in colorbrightness images).

\section{Visual features of the transformation of monochrome images (under the influ- ence of noise)}

Consider the monochrome images from table 11.

1) Sinusoid. With increasing noise level, the angle between the two lower "beams" increases, while the angle between the two upper "beams" decreases. The lower two "rays" become more prolonged, and the lateral lower ones increase in size and become lighter.

2) Radio pulse. With an increase in the noise level, there is a general tendency to transform the figure into a symmetrical, equilateral "star" with more rounded "rays" than the standard. When noise is added, the lower "beam" is split into two components.

3) The sequence of triangular pulses. With an increase in the noise level, the length of the "rays" is leveled, they become lighter, thinner and are grouped in pairs from above, from below, to the right and the left. In other words, the angle between the pairs of rays from above, below, to the right and the left, decreases.

4) Dirichlet function. With an increase in the noise level, the pairs of upper and lower "rays," simultaneously with the lower ones, increase and become lighter. Lateral upper "rays" do not change.

5) A sequence of rectangular pulses. With increasing noise levels, the lower and upper pairs of "rays" grow. "Rays" located between the side and bottom merge with the bottom.

\section{Visual features of the transformation of monochrome images (under the influ-} ence of noise)

Let us consider the color-brightness difference images from table 11.

1) Sinusoid. At a low noise level, a smoothed shape of the image is observed in which colors are concentrated in certain areas and do not mix. In this case, red color prevails. With an increase in the noise level (average level), the separation of color regions and their asymmetric superposition on each other occurs. When the image is very noisy, the scanning rays become more "sharp." With increasing noise levels, cool colors (green and blue) begin to dominate.

2) Radio pulse. At a low noise level, the image represents symmetrical "star" with a large number of identical "rays," with a predominance of red. With an average noise level, the amount of "red rays" is halved. The "shortened rays" of yellow-green color begin to occupy a large area of the image. With a high noise level, only four large "bright red beams" remain in the "star." Short green rays occupy almost the entire central region.

3) The sequence of triangular pulses. At a low noise level, the image represents a "star" with the two longest and brightest "red rays" in the upper part. With increasing noise, the angle between the position of the "red rays" increases, as well as the width of all the "rays"; they merge into one common color-brightness form.

4) Dirichlet function. At a low noise level, a pair of upper "red rays" and two pairs of the side "orange rays" are noticeable. The lower half of the "star" is colored green. As the noise level increases, the lower half of the image undergoes small changes, while the angle between the upper rays decreases, they shorten and change color from red to orange. At the same time, the upper "orange rays" significantly increase in size and repaint mainly in red. The lower side "orange rays" are shortened and turn green.

5) A sequence of rectangular pulses. With a low noise level in the upper part of the "star," a pair of the longest "rays" is observed, with a predominance of red. The four lateral upper "beams" are grouped in pairs and painted mainly yellow. The rays at the bottom of the image are shorter than at the top and are painted in green, blue, and indigo. With an increase in the noise level, a change in the lateral upper "yellow rays" is noticeable, namely: two of them increase, redden and align with the upper ones in length; the other two, located below, are shortened and change color to green. Simultaneously with these changes, the "rays" located in the lower part of the "star" increase in size, acquiring a predominantly yellow-green color. 


\section{Findings}

The paper analyzes the applicability of the method of visualization of multidimensional data to the monitoring of digital electronic signals. The resulting cognitive images can improve the ergonomic qualities of the interfaces of ground-based space stations and increase the efficiency of operators. The main advantages of the developed methods of graphic support are as follows: visual interpretation and reliability of signal control, ease of perception of full flows of information in real-time, and the ability to quickly determine states. In the final part of the paper, we summarize the results of the analysis of the developed cognitive images to help the operators of the GS CIS.

THIS work was financially supported by the RUSSIAN FEDERAL PROPERTY FUND (PROJECTS No. 18-37-00037, No. 18-07-00014).

\section{References}

1. Bezruk V.M., Ivanenko S.A. Detection and recognition of signals under conditions of hight a priori uncertainty in the tasks of radio monitoring. - Control, navigation and communication systems. Academic journal, 2018, No.2, pp.135-141. (in Russian)

2. O'Shea T. J., West N.; Vondal M.; Clancy T. Ch. Semi-supervised radio signal identification. - 19th International Conference on Advanced Communication Technology. - IEEE, 2017. - 5 p. DOI: 10.23919/ICACT.2017.7890052.

3. Zha X., Peng H., Yang S. A Deep Learning Framework for Signal Detection and Modulation Classification. - Sensors, MDPI, 2019. - 21 p. DOI: 10.3390/s19184042.

4. Emelaynova J.G.. Development of cognitive representation methods for real time dynamic systems. - Artificial Intelligence and Decision Making, 2016, No.3, pp.21-30. (in Russian)

5. Cole W.G. Medical cognitive graphics. - CHI '86: Proceedings of the SIGCHI Conference on Human Factors in Computing Systems, April 1986, pp.91-95.

6. Zenkin A. A. Cognitive computer graphics. - M.: Science, 1990. - 192 p. (in Russian)

7. Pospelov D.A. Cognitive Graphics - a window into the new world. - Software products and systems, 1992, No.2, pp.4-6. (in Russian)

8. Bashlykov A.A. Computer information systems for intelligent support of NPP operators. - M.: OJSC "RRIOMEOGI”, 2016. - 520 p. (in Russian)

9. Burdayev M.N. Hodographs and the equation of flight in a central gravitational field. Software Systems: Theory and Applications, 2012, Vol.3, No.3(12), pp.79-92. (in Russian)

10. Yankovskaya A.E., Yamshanov A.V. Intelligent learning-testing systems using cognitive technologies. - XII All-Russian Meeting on RMPM-2014 Management: Works. - M.: V.A. Trapeznikov Institute of Control Sciences of RAS, 2014, pp. 4183-4191. (in Russian)

11. Grishin V.G. The figurative analysis of experimental data. - M.: Science, 1982. - 237 c. (in Russian)

12. Zinoviev A. Yu. Visualization of multidimensional data. Krasnoyarsk State University Press Publ, 2000. - 180 p. (in Russian)

13. Novoselov Yu.V. Diagnostics of technological object using fractal cognitive image. Scientific and technical sheets of SPbSPU. Informatics. Telecommunications. Management. SPb: SPbPUPG, 2012, No.2(145), pp. 119-122. (in Russian)

14. Kieras D.E. A Guide to GOMS Model Usability Evaluation using GOMSL and GLEAN4. - Electrical Engineering and Computer Science Department University of Michigan, 2006. $-72 \mathrm{p}$.

15. Höllt T. Visual Workflows for Oil and Gas Exploration: Ph.D. Thesis. Thuwal. 2013. $153 \mathrm{p}$.

16. Fischer F. Visual Analytics for Situational Awareness in Cyber Security. PhD thesis, Univ. Konstanz, 2016. - 220 p.

17. Lyons R.G. Digital Signal Processing, 2th edition, English translation. - M.: LLC "Binom-Press”, 2006. - 656 p. (in Russian) 
18. Ifeachor E.C., Jervis B.W. Digital Signal Processing: A Practical Approach. - Pearson Education, 2002. - 933 p.

19. Sloka V. K. Problems of radar signal processing. - M: "Sov. radio", 1970, 256 p. (in Russian)

20. Baskakov, S. I. Radio circuits and signals, 2th edition, M.: "Higher school”, 1987, 446 p. (in Russian)

21. Zagoruiko N. G. Applied methods of data and knowledge analysis, Novosibirsk: "IM SB RAS", 1999, 270 p. (in Russian)

22. Zhvalevsky O. V. Selection of informative features: problem statement and method of its solution. - Transactions SPIRAS. Issue. 4. - SPb.: "Science", 2007, pp.416-426. (in Russian)

23. Khachumov M. V. Distances, metrics, and cluster analysis. - Artificial Intelligence and Decision Making, 2012, No.1, pp.81-89. (in Russian)

24. Khachumov M. V. Models of the Representation and Clustering of Semistructured Information. - Scientific and Technical Information Processing, 2015, Vol. 42, No. 5, pp. 388396.

25. Derczynski L. Complementarity, F-score, and NLP Evaluation // Proceedings of the Tenth International Conference on Language Resources and Evaluation (LREC'16). European Language Resources Association (ELRA) (Portorož, Slovenia, May, 2016), 2016, pp.261-266. 